\title{
Performance of Acute Flaccid Paralysis surveillance in Bauchi State, Nigeria, 2016
}

\author{
Luka M. Ibrahim*, Adamu Ningi and Jalal-Eddeen Saleh
}

Disease prevention and control, World health organization, Jos, Nigeria

\section{Objective}

To identify and address gaps in acute flaccid surveillance for polio eradication in Buchi state

\section{Introduction}

Poliomyelitis a disease targeted for eradication since $1988^{1}$ still pose public health challenge. The Eastern Mediterranean and African Regions out of the six World Health Organization (WHO) Regions are yet to be certified polio free ${ }^{2}$. The certification of the WHO Africa region is largely dependent on Nigeria, while the WHO Eastern Mediterranean is dependent on Pakistan and Afghanistan. Surveillance for acute flaccid paralysis (AFP) is one of the critical elements of the polio eradication initiative. It provides the needed information to alert health managers and clinician to timely initiate actions to interrupt transmission of the polio disease and evidence for the absence of the wild polio virus. ${ }^{3,4}$ One of the core assignments of the certification committee in all regions is to review documentation to verify the absence of wild poliovirus. ${ }^{5}$ Good and complete documentation is the proxy indication of the quality of the system while poor documentation translates to possibilities of missing wild poliovirus in the past. We evaluated the performance of the AFP surveillance system in Bauchi, which is among the 11 high risks states for wild polio virus in Nigeria to identify and address gaps in the surveillance system.

\section{Methods}

We conducted a cross-sectional study in Bauchi State. We assessed the material and documentations on AFP surveillance in eighteen of the twenty Local Government Areas (LGAs). We assessed the knowledge of the clinician at focal and non-focal sites on case definition of AFP, the number and method of stool specimen collection to investigate a case and types of training received for AFP surveillance. We verified AFP case investigations for the last three years: The caregivers (mothers) were interviewed to authenticate the reported information of AFP cases, the method used for stool specimen collection and feedbacks. Community leaders' knowledge on AFP surveillance was also assessed. Data was entered and analyzed in excel spread sheet.

\section{Results}

Review of the expected deliverables of 18 out of the 20 LGA disease surveillance and notification officers (DSNO) revealed that only $2(11 \%), 5(28 \%), 6(33 \%)$ and $7(39 \%)$ had evidence of polio outbreak investigation, supervisory reports, minutes of meeting and surveillance work plan respectively. Of the 31 AFP cases investigated, correct and complete information was 39\% for birth day, $26 \%$ for birth month of the child, $23 \%$ for date of onset of paralysis and $23 \%$ for date of investigation. Contacts of informants, AFP 001-3 were deficient in the focal and non-focal sites. The non-focal also lacked guidelines for integrated disease surveillance and response (IDSR) and terms of reference for surveillance focal person.

Knowledge of case definition of AFP was $71 \%$ and $30 \%$ among clinician at the focal and non-focal sites, respectively and $88 \%$ and $55 \%$ for method of stool collection among clinician at focal and nonfocal sites. Among the 38 care givers (mothers) interviewed $16(42 \%)$ did not remember the day or month the investigation for the AFP was conducted, $36(95 \%)$ gave the correct number of stool samples, $15(40 \%)$ mentioned that the stool samples were collected 24 hours apart and only $12(32 \%)$ received feedbacks. Majority (79\%) of the community leaders interviewed were aware of AFP and knew that stool was the specimen for investigation of the AFP but $21 \%$ did not know whom to report a case of AFP in their community

\section{Conclusions}

Our study revealed knowledge and documentations gaps in AFP surveillance for certification of polio-free in Nigeria. The state ministry of health and the WHO consultants in the polio eradication unit should update the knowledge of the health care workers at the operational levels on AFP surveillance. The state ministry of health and the WHO consultants should also provide all essential documents required for quality AFP surveillance and ensure their judicious use.

\begin{tabular}{lllll}
\multicolumn{5}{l}{ Knowledge of respondents on AFP surveillance } \\
\hline Knowledge grade & \multicolumn{5}{l}{ Case definition of AFP } & \multicolumn{2}{c}{ Stool sample collection } \\
& Focal sites & Non-focal sites & Focal sites & Non-focal sites \\
Very poor & $15(17.6 \%)$ & $14(32.6 \%)$ & $7(8.2 \%)$ & $6(14.0 \%)$ \\
Poor knowledge & $10(11.8 \%)$ & $12(27.9 \%)$ & $3(3.5 \%)$ & $9(20.9 \%)$ \\
Good knowledge & $30(35.3 \%)$ & $9(20.9 \%)$ & $28(32.9 \%)$ & $10(23.30 \%)$ \\
Very good & $30(35.3 \%)$ & $8(18.6 \%)$ & $47(55.3 \%)$ & $18(41.9 \%)$ \\
\multicolumn{1}{c}{ Total } & $85(100.0 \%)$ & $43(100.0 \%)$ & $85(100.0 \%)$ & $43(100.0 \%)$
\end{tabular}

\section{Keywords}

Poliomyelitis; Acute Flaccid Paralysis; Surveillance; Eradication,

\section{Acknowledgments}

We acknowledge the Bauchi state Primary Health Care Development Agency (NPHCDA) for supporting the study

\section{References}

1. World Health Assembly. Global Eradication of Poliomyelitis by the Year 2000. Geneva, Switzerland: World Health Organization; 1988. Resolution WHA 41.28.

2. Polio Global Eradication Initiative. 2014

3. World Health Assembly. Global Eradication of Poliomyelitis by the Year 2000. Geneva, Switzerland: World Health Organization; 1988. Resolution WHA 41.28.

4. Chandrakant Lahariya Global eradication of polio: the case for "finishing the job" Bull World Health Organ vol.85 n.6 Genebra Jun. 2007.

5. World Health Assembly. Global Eradication of Poliomvelitis by the Year 2000. Geneva, Switzerland: World Health Organization; 1988. Resolution WHA 41.28.

\section{*Luka M. Ibrahim}

E-mail: lukaimangveep@yahoo.com 